\begin{tabular}{|c|l|}
\hline Title & $\begin{array}{l}\text { Mechanical fabrication of metal nano contacts showing conductance quantization under electrochemical potential } \\
\text { control }\end{array}$ \\
\hline Author(s) & Kiguchi, Manabu; Konishi, Tatsuy a; Miura, Shinichi; Murakoshi, Kei \\
\hline Citation & $\begin{array}{l}\text { Physica_E: Low-dimensional Systems and Nanostructures, 29(3-4), 530-533 } \\
\text { https://doi.org/10.1016/.physe.2005.06.020 }\end{array}$ \\
\hline Issue Date & 2005-11 \\
\hline Doc URL & http://hdl.handle.net/2115/8610 \\
\hline Type & article (author version) \\
\hline File Information & PhysicaE_v29p530.pdf \\
\hline
\end{tabular}

Instructions for use 


\title{
Mechanical fabrication of metal nano contacts showing conductance quantization under electrochemical potential control
}

\author{
Manabu Kiguchi ${ }^{\mathrm{a}}$, Tatsuya Konishi ${ }^{\mathrm{a}}$, Shinichi Miura ${ }^{\mathrm{a}}$ and Kei Murakoshi ${ }^{\mathrm{a}, \mathrm{b}^{*}}$ \\ ${ }^{a}$ Division of Chemistry, Graduate School of Science, Hokkaido Univ., Kita 10 Nishi 8, Sapporo, Hokkaido, Japan \\ ${ }^{b}$ PRESTO, Japan Science and Technology Corporation, Toyonaka, Osaka 560-8531, Japan
}

\begin{abstract}
We have mechanically fabricated $\mathrm{Ni}$ and $\mathrm{Cu}$ nano constrictions in solution, and studied their electrical conductance under the electrochemical potential control. Conductance quantization can be observed with both metals. This is the first observation of the conductance quantization behavior for non-gold metal nano constrictions, which are mechanically fabricated in solution at room temperature. The conductance of $\mathrm{Cu}$ was quantized in units of $G_{0}\left(=2 e^{2} / h\right)$, and a sharp $1 G_{0}$ peak is observed in the conductance histogram. For $\mathrm{Ni}$, a conductance plateau showed a slightly negative slope, and a broad peak at $1 \sim 1.5 G_{0}$ was observed in the histogram. The conductance quantization behavior was discussed by comparing the result of nano constrictions fabricated in non-solution condition, with those fabricated by an electrochemical method. It is suggested that a certain atomic configuration was stabilized in solution under the electrochemical potential control.
\end{abstract}

PACS: 73.23.-b, 73.40.Jn, 73.40.Cg

Keywords: metal nano constrictions, quantized conductance, electrochemical potential

\section{Introduction}

Metal nano structures are interesting not only because they promise various new applications, but also because they exhibit novel quantum phenomena. One of the current topics of metal nano structures is conductance quantization. Electrical conductance through a thin metal wire in an atomic scale can be expressed by $G=2 e^{2} / h \sum T_{i}$ where $T_{i}$ is the transmission probability of the $i$-th conductance channel and $G_{0}$ $=2 e^{2} / \mathrm{h}$ is the unit of quantum conductance [1].

While most of previous studies have been done in ultra high vacuum (UHV) at low temperature, metal nano structures and their quantum conductance behavior in solution have recently attracted wide attention [2-4]. Figure 1 shows the schematic view of the electron flow through a conduction channel in solution. Fermi energy of the electron is strictly defined by the electrochemical potential. Furthermore,

\footnotetext{
*Corresponding author. Fax: +81-11-706-4810 E-mail address: kei@ sci.hokudai.ac.jp
} 
adsorption or desorption of molecules and ions can be also controlled by the electrochemical potential. These facts make possible to fabricate novel metal nano structures which are not present in UHV, and to control their structures and physical properties by the electrochemical potential. Recently, we proved that clear conductance quantization for $\mathrm{Pd}$ nano constrictions can be observed by the careful control of Pd deposition and dissolution. It should be noteworthy that conductance quantization was not observed in $\mathrm{UHV}$ at room temperature at $\mathrm{Pd}$ nano constrictions [4,5].

To study quantized conductance of metal nano constrictions, several thousands of repetitive experiments are usually needed. These requirements can be met by mechanical methods using a scanning tunneling microscope (STM) or the mechanically controllable break junction technique. In solution, on the other hand, electrochemical methods have been usually used for electrochemically deposited metals. Compared to the mechanical method, the electrochemical method needs long time to fabricate one nano constriction (typically $\sim 10 \mathrm{~min}$ ), and thus, most of previous studies have discussed the conductance quantization behavior with only limited set of measurements (typically 100). Further investigations are needed for quantitative discussion on quantized conductance in solution.

In the present study, we have fabricated $\mathrm{Ni}$ and $\mathrm{Cu}$ nano constrictions with the mechanical method, and studied their conductance. Nickel is a

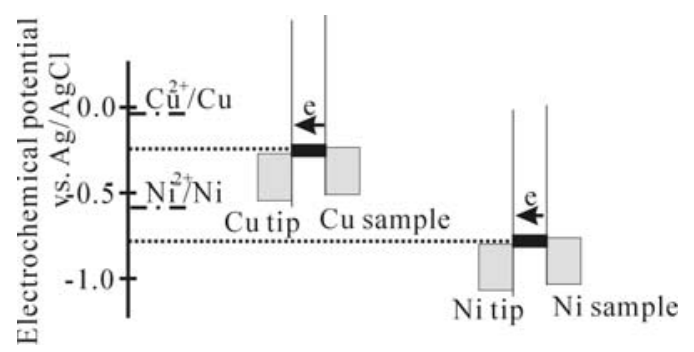

Fig. 1: A schematic view of the electron flow through a conduction channel. The dot-dashed and dotted lines indicate the equilibrium potential of electron chemical reaction $\mathrm{M}^{2+}+2 \mathrm{e}^{-} \rightarrow \mathrm{M}$ and the potential applied in the present studv.
$3 \mathrm{~d}$ transition metal, while $\mathrm{Cu}$ is a noble metal. We compared the quantized conductance of $\mathrm{Ni}$ with $\mathrm{Cu}$. Furthermore, the result was compared with that in UHV at ultra low temperature, and that with electrochemical method.

\section{Experimental}

Figure 2 (a) shows the block diagram of the conductance measurement system for the metal nano constrictions in solution. A signal access module provides access to every input and output signal between the controller and the microscope. A personal computer (PC) controls the STM tip's movement and monitors the electric current through the nano constrictions using this signal access module. To monitor the electric current, we use an analog-to-digital converter (InstruNet) installed in a PC. The electrochemical potential of the nano constriction is controlled using a potentiostat (Pico-Stat, Molecular Imaging Co.)

Fig. 2 (b) shows the schematic view of the experimental setup. The experiments were performed in an electrochemical cell mounted in a chamber that was filled with high purity $\mathrm{N}_{2}$ gas to reduce contamination. The tip was made of a gold (diameter $\sim 0.25 \mathrm{~mm}$ ) wire coated with wax to eliminate ionic conduction. The substrate was $\mathrm{Au}(111)$ prepared by flame annealing and

(a)

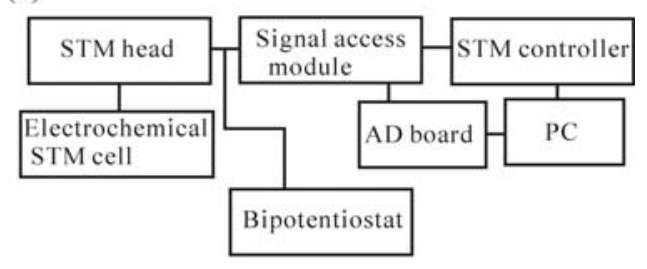

(b)

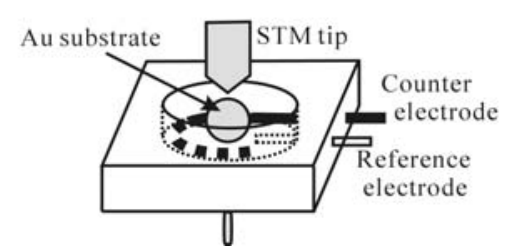

Fig. 2: (a) A block diagram of the conductance measurements system for metal nano constrictions. (b) A schematic view of the electrochemical STM cell. 
quenching method. The electrochemical potentials of the $\mathrm{Au}$ substrate and the tip were controlled with respect to the $\mathrm{Ag} / \mathrm{AgCl}$ reference electrode. A $0.50 \mathrm{~mm}$ diameter $\mathrm{Pt}$ wire was used as counter electrode. The electrolyte was a $1 \mathrm{mM} \mathrm{CuSO}_{4}$. $5 \mathrm{H}_{2} \mathrm{O}$ with $50 \mathrm{mM} \quad \mathrm{H}_{2} \mathrm{SO}_{4}$ for $\mathrm{Cu}$ nano constrictions, while it was a mixcture of $10 \mathrm{mM}$ $\mathrm{NiSO}_{4}, 10 \mathrm{mM} \mathrm{H}_{3} \mathrm{BO}_{3}$, and $1 \mathrm{mM} \mathrm{H}_{2} \mathrm{SO}_{4}$ for $\mathrm{Ni}$.

The equilibrium potential of electrochemical reaction $\mathrm{M}^{2+}+2 \mathrm{e}^{-} \rightarrow \mathrm{M}$ is $-600 \mathrm{mV}$ for $\mathrm{Ni}$, and $40 \mathrm{mV}$ for $\mathrm{Cu}$ in the present experimental condition assuming the Nernst equation (see Fig. 1). Both the $\mathrm{Au}$ tip and the $\mathrm{Au}(111)$ substrate were kept at an electrochemical potential of - 800 $\mathrm{mV}$ for $\mathrm{Ni}$, and $-350 \mathrm{mV}$ for $\mathrm{Cu}$. At this potential, the metals were electrochemically deposited on both the Au substrate and the tip. Then metal nano constrictions were created as the following manner. The feedback loop started by driving the STM tip into contact with the substrate. Once the contact was fully established as the conductance between the tip and the substrate reached a preset value (i. e. $4 G_{0}$ ), the STM tip was pulled out of the contact at a typical rate of $50 \mathrm{~nm} / \mathrm{s}$ with feedback off. During the pulling process, a Ni or $\mathrm{Cu}$ nano constriction was formed between the tip and the substrate. The same procedure was repeated over 3000 times. Conductance was calculated based on the potential difference between the tip and the substrate $(20 \mathrm{mV})$ and the observed current in the wire.

\section{Experimental results}

Figure 3 shows the typical conductance traces of the $\mathrm{Ni}$ and $\mathrm{Cu}$ nano constrictions during breaking. The electrochemical potential was -800 $\mathrm{mV}$ for $\mathrm{Ni}$ and $-260 \mathrm{mV}$ for $\mathrm{Cu}$. As a general tendency, the changes occured in a stepwise, rather than a continuous, fashion. Immediately prior to termination of the contacts, we frequently observed plateaus with the conductance of $1 G_{0}$ for $\mathrm{Cu}$, and 1-1.5 $G_{0}$ for Ni. Figure 4 shows the conductance histogram of the $\mathrm{Ni}$ and $\mathrm{Cu}$ nano constriction. A well-defined $1 G_{0}$ peak is observed in the histogram for $\mathrm{Cu}$, while a broad 1-1.5 $G_{0}$ peak is shown for Ni. It should be noticed that this

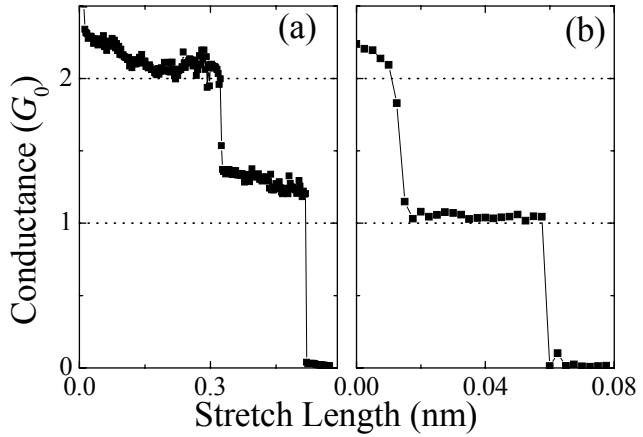

Fig. 3: Typical conductance traces of (a) Ni nano constrictions at electrochemical potential of $-800 \mathrm{mV}$, and (b) $\mathrm{Cu}$ nano constrictions at $-250 \mathrm{mV}$.

is the first observation of the conductance quantization behavior for non-gold metal nano constrictions, which are mechanically fabricated in solution at room temperature.

Here we compare the conductance quantization behavior in solution and that in nonsolution condition (e.g. air and UHV). A welldefined $1 G_{0}$ peak was also observed in the conductance histogram of $\mathrm{Cu}$ nano constrictions in non-solution condition at room temperature [7]. For Ni, a broad 1-1.5 $G_{0}$ peak appeared in the conductance histogram in UHV at ultra low temperature [8]. Whereas, the histogram was entirely featureless at room temperature, individual conductance curves presented welldefined steps [9]. This temperature dependence can be discussed in terms of fluctuation of the

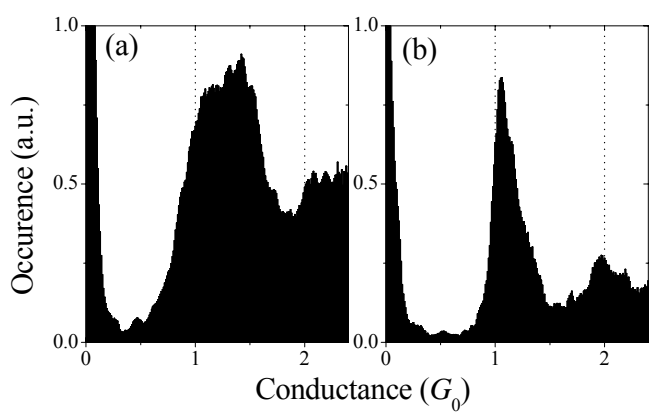

Fig. 4: Conductance histograms of (a) $\mathrm{Ni}$ and (b) $\mathrm{Cu}$ nano constrictions. 
environment. At room temperature, the thermal fluctuation of the atoms prevent the long-term stability of the nano contact, then certain atomic configurations are not preferentially formed. At low temperature, atomic size contacts can be held stable for rather long time. Therefore, the atomic configuration of the nano constriction would change into a thermally stable one, leading to appearance of a correspondig conductance peak in the histogram.

In solution, the fluctuation of environment seems to be much larger than in non-solution condition. However, a peak is clearly observed in the conductance histogram of the $\mathrm{Ni}$ nano constrictions in the present study. The histogram is similar to that obtained in UHV at ultra low temperature. Under the electrochemical potential control in solution, ions $\left(\mathrm{H}_{3} \mathrm{O}^{+}\right)$or $\mathrm{H}_{2} \mathrm{O}$ molecules would adsorb on the metal nano constriction, and a certain atomic configuration would be stabilized. Furthermore, the electronic state of the metal nano constrictions was well defined. Therefore, the conductance quantization behavior in solution was similar to that in UHV at low temperature.

Finally, we compare the present mechanical method with the electrochemical method. In the previous study, we have studied the conductance quantization behavior of the Ni nano constrictions fabricated by electrochemical deposition or dissolution. A clear and stable conductance plateau at $1 G_{0}$ was observed, and the conductance histogram exhibited a well-defined peak at $1 G_{0}$. In the case of electrochemical method, there is little strain in the metal nano constriction, and the inter atomic distance would be an energetically favorable one during the formation of a single atom contact. Therefore, the conductance histogram exhibites a single peak originating from this stable atomic configuration. On the other hand, the mechanical method fabricates nano constrictions by pulling the metal constrictions. Elastic deformation of the nano contact produces a slight increase in the inter atomic distance. The dependence of the conductance on the inter atomic distance varies with metals [10,11]. For simple metal like $\mathrm{Au}$ and $\mathrm{Cu}$, the conductance does not change with inter atomic distance, while the elongation of the contact induces a decrease in the conductance for non-s metals. Therefore, the change in inter atomic distance changeed the conductance for non-s metal $\mathrm{Ni}$, resulted in appearance of a plateau with a slightly negative slope, and a broad peak in the conductance histogram.

\section{Conclusion}

The conductance quantization behavior has been studied for the $\mathrm{Ni}$ and $\mathrm{Cu}$ nano constrictions, which were mechanically fabricated in solution. A clear $1 G_{0}$ peak was observed in the conductance histogram for $\mathrm{Cu}$, while a broad 1-1.5 $G_{0}$ peak was observed for Ni. We discussed the character of the quantized conductance in solution, and the difference between the electrochemical method and the mechanical method.

\section{Acknowledgement}

This work was partially supported by a Grantin-Aid for Scientific Research from MEXT.

\section{References}

[1] N. Agrait, A.L. Yeyati, and J.M. van Ruitenbeek, Physics Reports 377 (2003) 81.

[2] C. Z. Li, A. Bogozi, W. Huang, and N. J. Tao, Nanotechnology 10 (1999) 221.

[3] C. Z. Li and N. J. Tao, Appl. Phys. Lett. 72 (1998) 894.

[4] J.L. Li, T. Kanzaki, K. Murakoshi, and Y. Nakato, Appl. Phys. Lett. 81 (2002) 123.

[5] A. Enomoto, S. Kurokawa, and A. Sakai, Phys. Rev. B 65 (2002) 125410.

[6] F. Elhoussine, S. Mátéfi-Tempfli, A. Encinas, and L. Piraux, Appl. Phys. Lett. 81 (2002) 1681.

[7] J. C. González, V. Rodrigues, J. Bettini, L. G. C. Rego, A. R. Rocha, P. Z. Coura, S. O. Dantas, F. Sato, D. S. Galvão, and D. Ugarte, Phys. Rev. Lett. 93 (2004) 126103.

[8] L. Costa-Kramer, Phys. Rev. B 55 (1997) R4875.

[9] C. Untiedt, D. M. T. Dekker, D. Djukic, and J. M. van Ruitenbeek, Phys. Rev. B 69 (2004) R81401.

[10] J. C. Cuevas, A. Levy Yeyati, and A. Martín-Rodero, Phys. Rev. Lett. 80 (1998) 1066.

[11] J. C. Cuevas, A. Levy Yeyati, A. Martín-Rodero, G. Rubio Bollinger, C. Untiedt, and N. Agrait, Phys. Rev. Lett. 81 (1998) 2990. 